\title{
Joint Flow Control and Link Scheduling in Hybrid Terrestrial-Satellite Wireless Backhauling Network
}

\author{
Musbah Shaat and Ana I. Pérez-Neira \\ Centre Tecnològic de Telecomunicacions de Catalunya (CTTC/CERCA) \\ Parc Mediterrani de la Tecnología, Av. Carl Friedrich Gauss 7, 08860 , Castelldefels-Barcelona, Spain. \\ Phone: +34 93 6452900, Fax: +34 93 6452901, Email:\{musbah.shaat,ana.perez\}@cttc.es
}

\begin{abstract}
In this paper, the problem of cross-layer design of the link scheduling and flow control is studied in hybrid terrestrial-satellite wireless backhauling network. Considering network limitations and requirements, the target is to minimize the traffic delivery time by deciding the active backhauling links that can transmit simultaneously. Additionally, the proposed scheme provides a way to utilize the satellite links efficiently in order to provide the required link failure resiliency and traffic off-loading capability. Simulation results quantify the benefits of using the satellite links in cases of link failure and traffic congestion.
\end{abstract}

Keywords-Cross-layer Design, Flow Control, Link Scheduling, Satellite Networks, Wireless Backhauling.

\section{INTRODUCTION}

Wireless backhauling provides an efficient alternative to complement the wired backhauling solution of leasing bundled copper wires or using optical fiber cables due to their easy and low cost deployment and maintenance. The wireless backhauling should provide high throughput and reliable service to the end users as the wired backhauling does. Additionally, the different data applications which may contain a large volume of traffic should be supported. Accordingly, appropriate protocols as well as advanced radio technologies should be applied to boost the capacity of theses wireless links.

In wireless backhauling system, achieving maximum throughput in the network is not an easy task as it is not a question of optimizing the transmission parameters only, but it also requires a cross-layer optimization among the different layers to handle intelligently the link scheduling and traffic demand over the scheduled links. Each backhauling node operates as a gateway access point to its associated access nodes and at the same time as a wireless router to other nodes' traffic. By an adequate link activation policy, concurrent transmission of the network links can be achieved in the network while maintaining the Signal to Interference plus Noise Ratio (SINR) above the expected receiving threshold, which increases the spectral efficiency and exploit the flexibility of the wireless network. On top of that, introducing additional satellite links to the conventional terrestrial wireless backhauling network offers additional Degrees of Freedom (DoFs) that should be explored by the network. The satellite backhauling links can provide additional capability to the wireless backhauling network to serve higher user demands. Accordingly, satellite links are not only used to increase the terrestrial links fault tolerance, but also in cases where traffic offloading is needed or in zones where terrestrial links have no coverage.
Medium Access Control (MAC) protocol design for ad-hoc multi-antenna network is studied widely in the literature, e.g. see [1], [2] and references therein. The schemes are based mainly on suboptimal heuristic algorithms. The techniques developed for ad-hoc networks are not suitable for backhaul mesh networks. This results mainly from the dissimilarity in the generated traffic flows between ad-hoc and backhauling mesh networks. In ad-hoc networks, the nodes exchange data between each others while in backhauling mesh networks, the different nodes deliver the generated traffic to/from the core network. Additionally, centralized entity coordinates resources distribution and admission control.

By solving the scheduling problem, the active links that can transmit simultaneously in the network are decided. Centralized scheduling to provide per flow Quality of Service (QoS) is investigated in [3] and [4] with and without spatial reuse, respectively. Additionally, a scheduling algorithm is developed in [5] to maximize the system utilization and achieve a proposed fairness objective that is linked with the actual traffic demand. In [6], the interference impact and the available DoFs in each node are considered using an interference aware tree construction routing algorithm. The scheduling is performed via heuristic algorithms. By considering the flow control problem, the amount of traffic that should be routed on the different active links is decided. [7], [8] showed that routing without any consideration of interference can lead to significant performance degradation in wireless networks.

The problem of joint flow control and scheduling in wireless mesh network is NP-complete problem [9]. In [10], the joint optimization problem in Time Division Multiple Access (TDMA)-based multi-hop wireless network with MIMO link is investigated. A constant factor approximation and heuristic algorithms are proposed. Distributed link scheduling and power control algorithm based on decomposing the system into multiple isolated MIMO broadcast subsystems is proposed in [11]. In [12], cross physical-link layers design of wireless network with MIMO enabled nodes under power constraints is studied. The non-linear problem is solved by decomposing the problem into several sub-problems which are solved in a distributed manner. Afterwards, heuristic algorithm is proposed to solve the same problem with lower computational complexity. In [13], joint optimal design of flow control and MAC scheduling with beamforming at the physical layer is considered. The dual decomposition method is used to decouple the flow control and scheduling sub-problems. In [14], joint optimization of flow control and scheduling is considered where the interfering links 


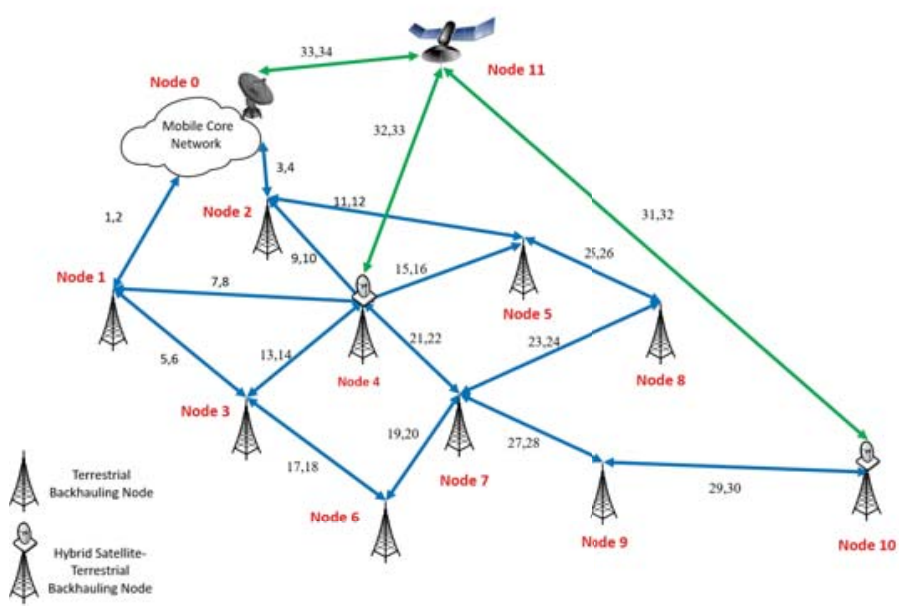

Fig. 1. Wireless Backhauling Network.

are determined according to the distance between the nodes. In [15], the routing and scheduling scheme is proposed to handle the traffic dynamics and traffic uncertainty.

The work in this paper is different from the already mentioned papers in that it considers the joint flow control and link scheduling in a Spatial Time Division Multiple Access (STDMA) based wireless backhaul network, where both terrestrial and satellite links are available in the system. The problem is solved by taking into account the use of the satellite links in cases of traffic offloading, link failure and remote access. However, the system can be configured to have all unrestricted usage of the satellite links. The rest of the paper is organized as follows. Section II provides details of the system model while Section III formulates the optimization problem. The optimization problem is solved in Section IV. Simulation results are provided and discussed in Section V. Finally, Section VI concludes the paper.

\section{SySTEM MODEL}

The system considered in this work is a multi-hop wireless backhaul network, where there are several backhauling nodes that serve multiple users in a given geographical area. Not all the nodes have direct connection to the core network and hence reach the core network through multi-hop links. Additionally, some nodes have hybrid terrestrial-satellite communication capabilities which enable them to reach the core network through satellite. The considered multi-hop network is depicted in Fig. 1.

The network is modeled as a directed graph $\mathcal{G}=\{\mathcal{N}, \mathcal{L}\}$ where $N=\{0,1, \cdots, N\}$ is the set of all backhauling nodes (vertices) $n$ equipped with multiple antennas. Node 0 represents the core network and $\mathcal{L}$ is the set of all available links (edges). A link exists between two nodes if they are in the transmission range of each other, i.e. $\mathcal{L}=\left\{l, d_{t(l), r(l)} \leq\right.$ $\left.T_{t(l)}, t(l) \in N, r(l) \in N, t(l) \neq r(l)\right\}$ where $d_{t(l), r(l)}$ is the distance between the transmitter on the $l^{\text {th }}$ link, $t(l)$, and the receiver on the $l^{t h}$ link, $r(l) . T_{t(l)}$ is the transmission range of the transmitting node $t(l)$. The incidence matrix of the graph
$\mathcal{G}$ is defined as follows

$$
I(n, l)= \begin{cases}1, & \text { if the node } n \text { is transmitting on } l, \\ -1, & \text { if the node } n \text { is receiving on } l, \\ 0, & \text { otherwise }\end{cases}
$$

Additionally, to indicate whether a given link is active or not in a given scheduling configuration, the following binary variable is defined

$$
a_{l}^{c}= \begin{cases}1 & \text { if the link } l \text { is active in c } \\ 0 & \text { otherwise, }\end{cases}
$$

where $c$ is a possible link scheduling configuration that includes all the active links, i.e. $c=\left\{a_{l}^{c}, \forall l \in \mathcal{L}\right\}$. We are considering the uplink scenario, where the traffic generated at the different nodes is transmitted to the core network. However, a similar approach can be used for the downlink case. Not all the links in the system can be active at the same time due to radio or interference constraints. Accordingly, the objective is to decide which link scheduling configuration should be active at a given time scheduling interval and decide at the same time the amount of flow that should be transmitted on the different active links of the selected scheduling configuration. Accordingly, STDMA is employed so that the time slots can be shared by simultaneous transmission whenever possible. In the following subsections, details of the radio and interference constraints are discussed.

\section{A. Radio Constraints}

The transceivers in the backhauling nodes are assumed to be working in half-duplex mode. Accordingly, any backhauling node can either transmit, or receive and suppress interference at the same time. This is usually called the primary conflict. Define $\mathcal{L}_{n}=\left\{\mathcal{O}_{n}^{+} \cup \mathcal{O}_{n}^{-}\right\}$to be the set of the links that are connected to the node $n$ where $\mathcal{O}_{n}^{+}$is the set of outgoing links of node $n$, i.e. $\mathcal{O}_{n}^{+}=\{l: I(n, l)=1\}$ while $\mathcal{O}_{n}^{-}$is the set of ingoing links to node $n$, i.e. $\mathcal{O}_{n}^{-}=\{l: I(n, l)=-1\}$. To satisfy the radio constraint, the links with no primary conflict in a given scheduling configuration $c$ should satisfy the following constraint

$$
a_{j}^{c}+a_{k}^{c} \leq 1 \forall n \in \mathcal{N}, j \in \mathcal{O}_{n}^{+}, k \in \mathcal{O}_{n}^{-} .
$$

Equivalently, (3) can be written as $\sum_{j \in \mathcal{O}_{n}^{+}, k \in \mathcal{O}_{n}^{-}} a_{j}^{c} a_{k}^{c}=$ $0, \forall n \in \mathcal{N}$. When multiple link transmission is not allowed, i.e. single transmission link per node, the half-duplex transmission constraint becomes $\sum_{l \in \mathcal{L}_{n}} a_{l}^{c} \leq 1 \forall n \in \mathcal{N}$.

A system with a full duplex Frequency Division Duplexing (FDD) capabilities can transmit and receive simultaneously and hence, this radio constraint is not applied. This is also valid if the system uses any In-Band Full-Duplex (IBFD) techniques.

\section{B. Interference Constraints}

The activation of the links does not depend only on the radio limitation, but also on the level of interference between the neighboring nodes. Accordingly, two interfering links should not be activated simultaneously. This type of conflict is called the secondary conflict. A scheduling configuration is the set of links that are free from both primary and secondary conflicts. As the interference cannot be eliminated completely while sharing network resources, minimum SINR guarantee 
on every active link is a possible way to decide the active links. The approach of optimizing the use of the available DoFs presented in [10] or the one that solves the problem of finding the transmit and receive performing weight [13], [16] can be applied. We assume that the system has sufficient resources and DoFs to use the different links and maintain the minimum required SINR on the links.

\section{PROBLEM Formulation}

We are interested in the problem of upstreaming the aggregated traffic generated by the different users in a given geographical area to the core network. A similar approach can be followed for the downstream as well. The target is to decide which scheduling configuration should be used at a given time, as well as the amount of traffic that should be transmitted on each link. The Multi-Commodity Flow (MCF) model is assumed here, considering that the queuing effect is negligible at each backhauling node and the aggregate traffic demand per cell is constant over the scheduling execution time. Let $f_{l}^{d}$ represents the amount of flow assigned to the $l^{\text {th }}$ link and corresponding to the commodity $d$. The generated traffic at each backhauling node is considered as a single commodity $s_{n}$. The flow conservation law should be satisfied to ensure that the sum of ingoing and outgoing flows belonging to the commodity $d$ at each relaying backhauling node is equal; which can be expressed mathematically as

$$
\sum_{l \in \mathcal{O}_{n}^{+}} f_{l}^{d}=\sum_{l \in \mathcal{O}_{n}^{-}} f_{l}^{d}, \forall n \in \mathcal{N} \backslash\{0, d\}, d \in \mathcal{N} \backslash\{0\} .
$$

Note that Node 0 and Node $d$ are excluded in (4) as they are representing the source and destination nodes. For each source backhauling node, the sum of the traffic should be equal to the amount of the traffic generated in the node as given in the following equation

$$
\sum_{l \in \mathcal{O}_{n}^{+}} f_{l}^{d}-\sum_{l \in \mathcal{O}_{n}^{-}} f_{l}^{d}=s_{d}, \forall d \in \mathcal{N} \backslash\{0\} .
$$

The sum of flows belonging to different commodities that are passing through a given link should not exceed the capacity of that link. Let $\lambda_{c}$ denotes the fraction of time in which the scheduling configuration $c$ is active and $\bar{C}$ to represent the number of all possible scheduling configurations, hence the effective MAC layer capacity is expressed as

$$
\sum_{d \in N, d \neq 0} f_{l}^{d} \leq \sum_{c \in \bar{C}} \lambda_{c} a_{l}^{c} x_{l}, \forall l \in \mathcal{L},
$$

where $x_{l}$ is the physical layer capacity which depends on the physical layer resources like power, bandwidth, interference, etc. The joint flow control and link scheduling problem with admissible traffic, i.e. $\sum_{c \in \bar{C}} \lambda_{c} \leq 1$, can be mathematically formulated as follows

$$
\begin{aligned}
& \min _{\lambda_{c}, f_{l}^{d}} U\left(s, \lambda_{c}, f_{l}^{d}\right) \quad \text { s.t. } \\
& \mathrm{C} 1: \sum_{l \in \mathcal{O}_{n}^{+}} f_{l}^{d}=\sum_{l \in \mathcal{O}_{n}^{-}} f_{l}^{d}, \forall n \in \mathcal{N} \backslash\{0, d\}, d \in \mathcal{N} \backslash\{0\} \\
& \mathrm{C} 2: \sum_{l \in \mathcal{O}_{n}^{+}} f_{l}^{d}-\sum_{l \in \mathcal{O}_{n}^{-}} f_{l}^{d}=s_{d}, \forall d \in \mathcal{N} \backslash\{0\} \\
& \mathrm{C} 3: \sum_{d \in N, d \neq 0} f_{l}^{d} \leq \sum_{c \in \bar{C}} \lambda_{c} a_{l}^{c} x_{l}, \forall l \in \mathcal{L} \\
& \mathrm{C} 4: f_{l}^{d} \geq 0 \\
& \mathrm{C} 5: \lambda_{c} \geq 0,
\end{aligned}
$$

where $C 1$ and $C 2$ satisfy the flow conservation law, while $C 3$ represents the MAC layer capacity constraints. $C 4$ and $C 5$ ensure positive values of both the amount of flow and the scheduling configuration time ratios.

$U\left(s, \lambda_{c}, f_{l}^{d}\right)$ represents the utility function which is selected in a way that captures the objective of both minimizing the scheduling time to deliver a fixed amount of traffic and at the same time to account for the cost of using the satellite links when compared with the terrestrial links. Accordingly, the utility function is formulated as

$$
U\left(s, \lambda_{c}, f_{l}^{d}\right)=\sum_{c=1}^{\bar{C}} \lambda_{c}+\Delta \sum_{l \in \mathcal{S}} \sum_{d \in N, d \neq 0} f_{l}^{d},
$$

where $\Delta$ is a positive constant that is multiplied by the sum of flows on the satellite links. $\mathcal{S}$ is the set that contains all the satellite links. Once an amount of flow is allocated to any satellite link, the utility function increases. However, the effect of this increment on the utility function is calibrated by selecting the value of $\Delta$. By considering this utility function and suitable value of $\Delta$, the satellite links are used in case the amount of traffic is not schedulable by the terrestrial nodes or in case of link failure that prevents a node from reaching the core network. Accordingly, taking advantage of the inclusion of higher capacity satellite links with continues availability and with no interference with the terrestrial links, and avoid using the satellite links when the terrestrial network is capable of scheduling the traffic. Similar utility function has not been considered before. One of the main contributions of this paper is to reveal the benefits of using the satellite backhanding in case of link failure and traffic congestion.

\section{Solution OF the Joint Flow Control And SCHEDULING PROBLEM}

To obtain the optimal solution for problem (7), all the scheduling configurations should be generated so that the problem is solved over all of them. Generating all the possible configurations is not practical as the number of configurations grows exponentially with the size of the network. Alternatively, column generation technique can be used to solve the problem over a subset of the possible scheduling configurations [13], [17]. This approach is widely applied in large sized linear programming setups. In general terms, the column generation method has two main parts, the master problem and the pricing one. The master problem consists of solving the original problem with a restricted scheduling configurations while the pricing problem incorporates the dual variables found by the master problem to find the new scheduling configuration (column) to be added to the initial restricted scheduling configurations. The process continues until no new/better scheduling configuration is added. Let $\overline{C_{T}} \subset \bar{C}$ represents a subset of the scheduling configurations, hence the master problem can be re-formulated mathematically as follows

$$
\begin{gathered}
\min _{\lambda_{c}, f_{l}^{d}} U\left(s, \lambda_{c}, f_{l}^{d}\right)=\sum_{c=1}^{\overline{C_{T}}} \lambda_{c}+\Delta \sum_{l \in \mathcal{S}} \sum_{d \in N, d \neq 0} f_{l}^{d} \\
\text { s.t. } \quad(\mathrm{C} 1),(\mathrm{C} 2),(\mathrm{C} 3),(\mathrm{C} 4),(\mathrm{C} 5) .
\end{gathered}
$$

To solve problem (9) by applying the dual composition technique, the dual problem associated with the primal prob- 
lem (9) can be written as

$$
\max _{\mu_{l} \geq 0, \forall l \in \mathcal{L}} g\left(\mu_{l}\right),
$$

where $\mu_{l}$ are the dual variables associated with the MAC layer capacity constraint and $g\left(\mu_{l}\right)$ is the dual function defined as follows

$$
\begin{gathered}
g\left(\mu_{l}\right) \triangleq \min _{f_{l}^{d} \geq 0, \lambda_{c} \geq 0} \mathcal{F}\left(\lambda_{c}, f_{l}^{d}, \mu_{l}\right) \\
\text { s.t. } \quad(\mathrm{C} 1),(\mathrm{C} 2),
\end{gathered}
$$

where $\mathcal{F}\left(\lambda_{c}, f_{l}^{d}, \mu_{l}\right)$ is the Lagrangian function given by

$$
\begin{aligned}
& \mathcal{F}\left(\lambda_{c}, f_{l}^{d}, \mu_{l}\right)=\sum_{\overline{\mathcal{C}_{T}}}^{\bar{C}}+\Delta \sum_{l \in S} \sum_{d \in \mathcal{N}, d \neq 0} f_{l}^{d} \\
& \quad+\sum_{l \in \mathcal{L}} \mu_{l}\left(\sum_{d \in \mathcal{N}, d \neq 0} f_{l}^{d}-\sum_{c \in \bar{C}_{T}} \lambda_{c} a_{l}^{c} x_{l}\right) .
\end{aligned}
$$

The Lagrangian function can be re-arranged as

$$
\begin{aligned}
& \mathcal{F}\left(\lambda_{c}, f_{l}^{d}, \mu_{l}\right)=\sum_{c=1}^{\overline{\mathcal{C}_{T}}} \lambda_{c}-\sum_{l \in \mathcal{L}} \mu_{l} \sum_{c \in \bar{C}_{T}} \lambda_{c} a_{l}^{c} x_{l} \\
& \quad+\Delta \sum_{l \in S} \sum_{d \in \mathcal{N}, d \neq 0} f_{l}^{d}+\sum_{l \in \mathcal{L}} \mu_{l} \sum_{d \in \mathcal{N}, d \neq 0} f_{l}^{d} .
\end{aligned}
$$

The first two terms contain the link scheduling variable $\lambda_{c}$ while the last two terms contain the flow control variable $f_{l}^{d}$. Accordingly, the dual function can be separated into two independent sub-problems. The link scheduling sub-problem can be formulated as

$$
\begin{aligned}
& \min _{\lambda_{c}} \sum_{c=1}^{\overline{\mathcal{C}_{T}}} \lambda_{c}-\sum_{l \in \mathcal{L}} \mu_{l} \sum_{c \in \bar{C}_{T}} \lambda_{c} a_{l}^{c} x_{l} \\
& \text { s.t. } \quad \lambda_{c} \geq 0, \forall c \in \overline{\mathcal{C}_{T}} \text {, }
\end{aligned}
$$

and the flow control sub-problem can be formulated as

$$
\begin{gathered}
\min _{f_{l}^{d} \geq 0} \Delta \sum_{l \in S} \sum_{d \in \mathcal{N}, d \neq 0} f_{l}^{d}+\sum_{l \in \mathcal{L}} \mu_{l} \sum_{d \in \mathcal{N}, d \neq 0} f_{l}^{d} \\
\text { s.t. } \quad(\mathrm{C} 1),(\mathrm{C} 2) .
\end{gathered}
$$

The subgradient method [18] is used to solve the dual problem with guaranteed convergence. After finding the optimal solution, i.e. $f_{l}^{d^{*}}$ and $\lambda_{c}^{*}$ of the dual function at a given dual point $\mu_{l}$, the dual variables at the $(i+1)^{t h}$ iteration are updated as follows

$$
\mu_{l}^{(i+1)}=\mu_{l}^{(i)}-\delta^{(i)}\left(\sum_{d \in \mathcal{N}, d \neq 0} f_{l}^{d}-\sum_{c \in \bar{C}_{T}} \lambda_{c} a_{l}^{c} x_{l}\right),
$$

where $\delta^{(i)}$ is the step size that can be updated according to the nonsummable diminishing step size policy [18].

Once the master problem is solved, the pricing problem is solved by ensuring that the reduced cost of any new scheduling configuration satisfies

$$
\min _{c \in \mathcal{C} \backslash \overline{\mathcal{C}}_{T}} 1-\sum_{l \in \mathcal{L}} a_{l}^{c} \mu_{l}<0
$$

If the resulting reduced cost is not negative or the obtained configuration set is already available in $\overline{\mathcal{C}_{T}}$, the obtained solution of the master problem is the optimal solution of the main problem. If the cost is negative, the obtained scheduling configuration is added to $\overline{\mathcal{C}_{T}}$ and the master problem is solved again.

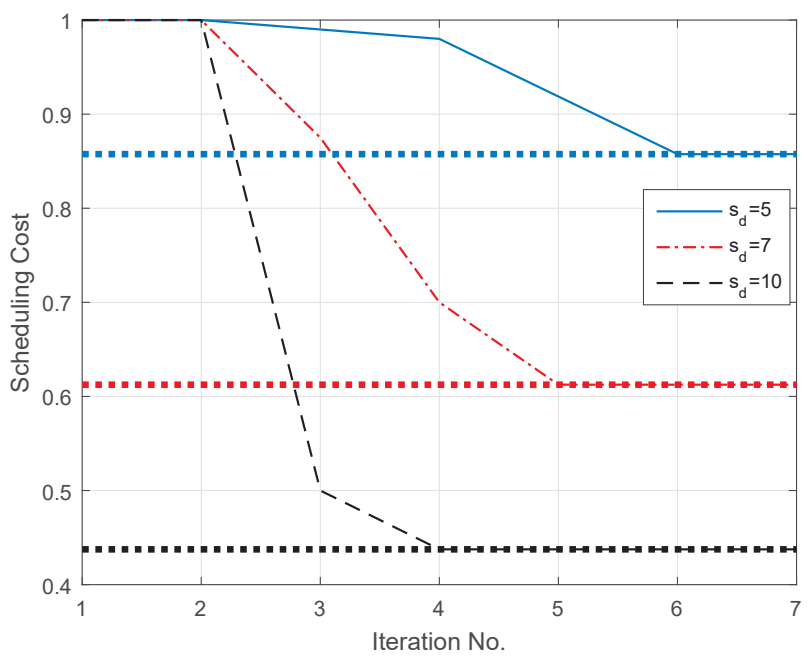

Fig. 2. Convergence of the column generation algorithm

To summarize the solution procedures, firstly a subset of the scheduling configurations is selected. Afterwards, the master problem is solved considering the restricted scheduling configuration set to find the dual variables related to the MAC layer capacity constraints. These dual variables are used to find a possible new scheduling configuration via solving the pricing problem. Depending on the reduced cost value and the existence of the obtained scheduling configuration in the subset used by the master problem, the algorithm decides whether the solution is found or a new iteration is required after adding the obtained scheduled configuration to the initial restricted subset.

\section{Simulation Results}

To show the performance of the proposed scheme, the topology depicted in Fig. 1 is considered where there are 11 nodes and 34 directional links. The proposed solution is not restricted to the simulated topology in Fig. 1 and can be applied to any hybrid terrestrial-satellite network. The numbers above the links refer to the link's number. The mobile core network is referenced as Node 0. Nodes 4 and 10 are assumed to be hybrid satellite-terrestrial nodes and connected to satellite (Node 11) while the rest of the nodes are assumed to be terrestrial only. The satellite node is forwarding the incoming traffic and has no generated traffic. We assume that the system has sufficient resources and DoFs to use these links and maintain the minimum required SINR on the links. All the nodes traffic demands as well as the channel capacities are normalized to $1 \mathrm{Mbps}$. We consider that the two satellite links have capacities of $160 \mathrm{Mbps}$ which is the double of the capacities of the terrestrial links. The algorithm performance is not depending on a specific selection of these parameters. We always consider the uplink streaming; however, the downlink streaming is similar and can be adopted accordingly. The starting set of scheduling configurations $\overline{\mathcal{C}}_{T}$ represents a simple TDMA scheme, i.e. only one link is active in every scheduling configuration.

First, we compare the performance of the proposed scheme at different iterations and check the convergence of the column generation method to the optimal solution. The optimal solu- 


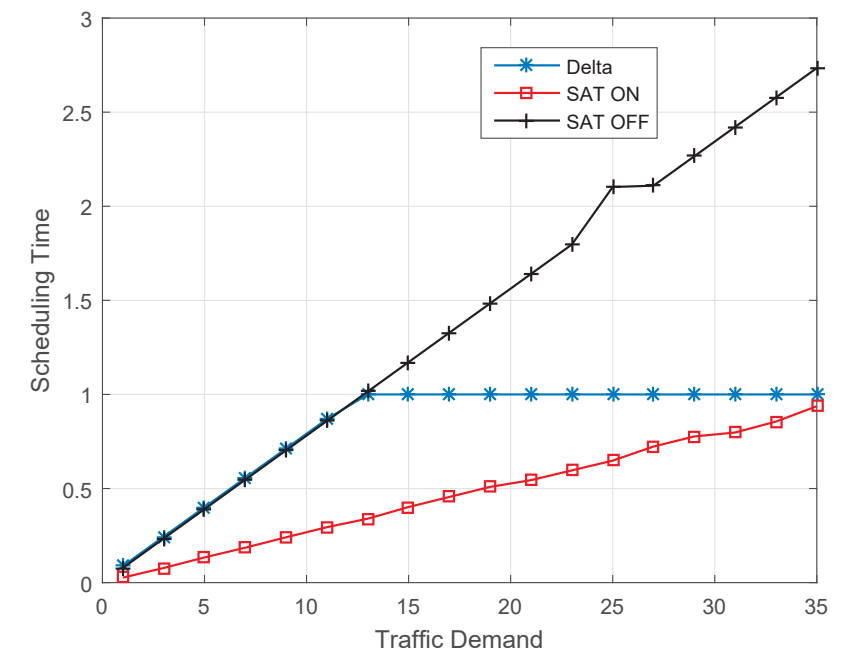

Fig. 3. Scheduling time vs. traffic demand per node with high $\Delta=1$.

tion is evaluated considering all the maximal transmission sets. The scheduling cost against the iteration number is plotted in Fig. 2 with $\Delta=1$ and different traffic demands. All the terrestrial nodes are assumed to have the same amount of generated traffic. It is revealed that the column generation technique is capable of achieving the optimal solution with few iterations. The dotted horizontal lines represent the optimal value for each traffic demand. It can be seen from the figure, that more scheduling cost is required when the traffic demand per node increases. This is due to the need of scheduling higher traffic and the use of the different scheduling configurations for an extended period of time.

The utility function in (8) has two main parts. The first part represents the scheduling cost, which is the summation of the fraction of time over which a given scheduling configuration is used. The second part consists of the multiplication of constant $\Delta$ by the traffic flow on the satellite links. Selecting high value of $\Delta$ enforces the system to avoid transmitting on satellite links unless the traffic is not schedulable by the terrestrial network, i.e. $\sum_{c \in \bar{C}} \lambda_{c} \leq 1$. Accordingly, this case enable the satellite usage only whenever necessary. Another case is considering a zero or close to zero $\Delta$. In this case, the system assumes that the satellite links are always available even for a small amount of traffic. The third case considers selecting a value for $\Delta$ that makes the system behave in an intermediate state between blocking the use of satellite if the terrestrial links are sufficient and enable the free use of the satellite links. The case of high value of $\Delta$ is used for the rest of simulations.

Fig. 3 depicts the scheduling cost of the network with high $\Delta$ where the proposed utility function is compared with networks with the cases of having always available satellite links and with no available satellite links. We referred to these cases by "SAT ON" and "SAT OFF", respectively. Additionally, we referred to the proposed scheme by "Delta". It can be noted that the scheduling time increases with the increment of the traffic per node and always the "SAT ON" scheme has the lowest scheduling cost. As expected, when the traffic is schedulable by the terrestrial nodes, the proposed utility function network acts as "SAT OFF". Once the traffic can not be schedulable by the terrestrial nodes, i.e. the scheduling cost

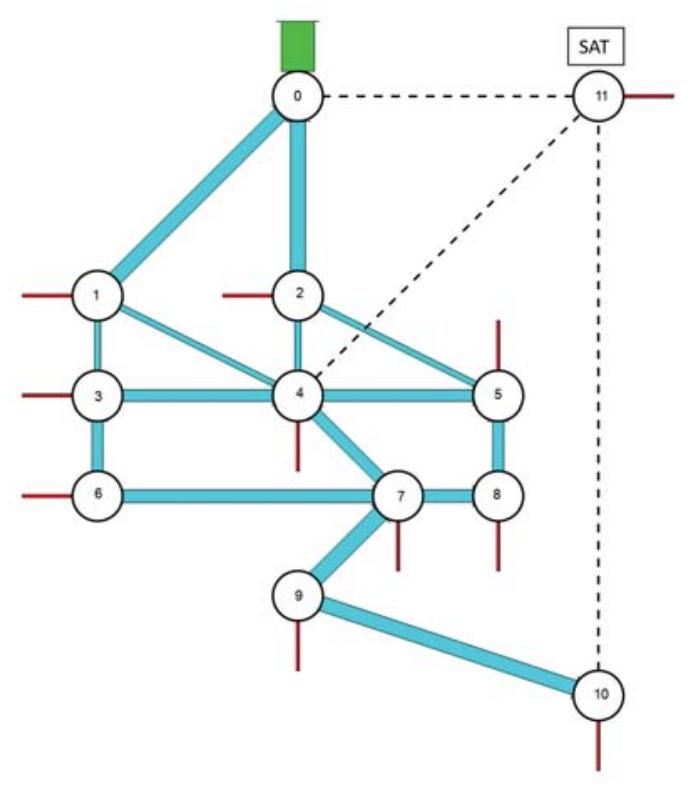

Fig. 4. Flow assignment before links failure between nodes 1,2 and the core and before congestion, $\sum_{c} \lambda_{c}=0.8750$.

is more than one, the satellite links are used with the terrestrial ones to schedule the traffic. The value of the scheduling cost is always one because the algorithm, by applying the proposed utility function, tries to reduce as much as possible the amount of traffic transmitted by the satellite links. Hence, it is not searching only for the scheduling configurations that enable reduced scheduling time but also that allow minimal traffic to flow over the satellite links.

To simulate the link failure, the amount of traffic demand per node is assumed to be $s_{d}=10$. Firstly, we assume that links between the Nodes 1,2 and the core network are failed. Accordingly, the nodes have to reach the core network through the satellite. Fig. 4 and Fig. 5 depict the flow assignment per link before and after the link failure respectively. The thickness of the lines represents the amount of flow on that link. The redcolored lines -lines entering the nodes- represent the generated traffic per nodes while the green one-line leaving the reference Node 0- represents the traffic received by the core network. Dashed lines represent available links with zero traffic.

For the link congestion simulation, the amount of traffic demand per node is assumed to be $s_{d}=10$ prior to congestion. It is assumed that the traffic generated at Node 4 is increased due to an event with $s_{4}=100$. This increment in the traffic limits the ability of the terrestrial links to schedule the traffic and hence, part of this traffic should reach the core network through the satellite link. Fig. 6 depicts the flow assignment per link after the congestion. The prior to congestion traffic assignment is already plotted in Fig. 4.

\section{CONCLUSION}

In this paper, the problem of allowing concurrent transmissions over the network links is addressed. The problem of cross-layer design of the flow control and link scheduling policies in hybrid terrestrial-satellite wireless backhaul network is treated with the objective of minimizing the time required to deliver the traffic. The problem is solved by taking into 


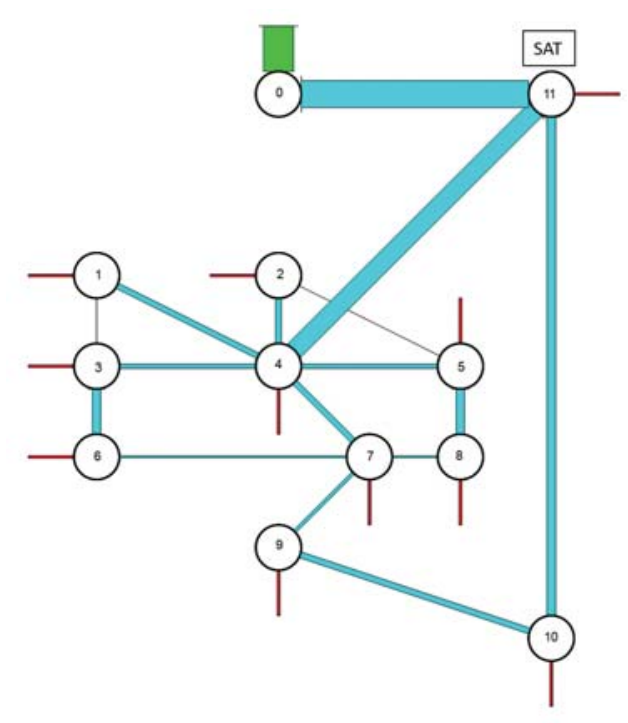

Fig. 5. Flow assignment after links failure between nodes 1,2 and the core, $\sum_{c} \lambda_{c}=0.4250$.

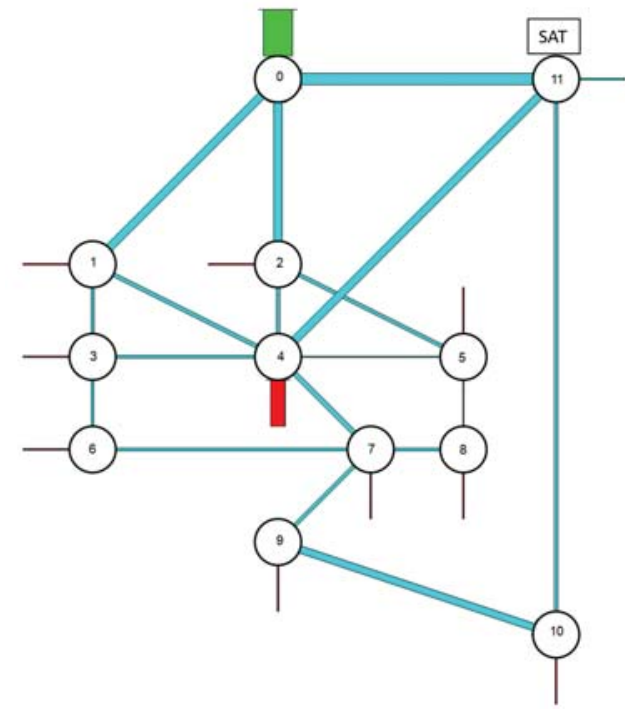

Fig. 6. Flow assignment after link congestion, $\sum_{c} \lambda_{c}=1$.

account the use of the satellite links only in cases of traffic offloading, link failure and remote access. The active links are selected based on the network requirements and limitations. As considering all the links activation possibilities is not practical, column generation technique is used to obtain the optimal solution with lower complexity and guaranteed convergence. The benefits of using the satellite links in cases of link failure and traffic congestion are revealed via simulations. We are working on developing a radio resource management framework in order to consider efficient utilization of the network resources. Additionally, incorporation of traffic differentiation aware algorithm in the system is being studied to take delay sensitive applications into consideration.

\section{ACKNOWLEDGMENT}

This work is supported by the European Commission in the framework of the H2020 SANSA (Shared Access Terrestrial-
Satellite Backhaul Network enabled by Smart Antennas) project (Grant agreement no.645047) and by the Spanish Government under project ELISA (TEC2014-59255-C3-1-R).

\section{REFERENCES}

[1] K. Sundaresan, R. Sivakumar, M. A. Ingram, and T.-Y. Chang, "Medium access control in ad hoc networks with MIMO links: optimization considerations and algorithms," IEEE Transactions on Mobile Computing, vol. 3, no. 4, pp. 350-365, 2004.

[2] X. Wang and J. Garcia-Luna-Aceves, "Embracing interference in ad hoc networks using joint routing and scheduling with multiple packet reception," Ad Hoc Networks, vol. 7, no. 2, pp. 460-471, 2009.

[3] H. Shetiya and V. Sharma, "Algorithms for routing and centralized scheduling to provide qos in IEEE 802.16 mesh networks," in Proceedings of the 1st ACM workshop on Wireless multimedia networking and performance modeling, 2005, pp. 140-149.

[4] H.-Y. Wei, S. Ganguly, R. Izmailov, and Z. J. Haas, "Interference-aware IEEE 802.16 WiMax mesh networks," in 2005 IEEE 61st Vehicular Technology Conference, vol. 5, 2005, pp. 3102-3106.

[5] M. Cao, V. Raghunathan, and P. Kumar, "A tractable algorithm for fair and efficient uplink scheduling of multi-hop wimax mesh networks," in 2006 2nd IEEE Workshop on Wireless Mesh Networks, 2006, pp. 93-100.

[6] Y. Xu, S. Wan, J. Tang, and R. S. Wolff, "Interference aware routing and scheduling in wireless backhaul networks with smart antennas," in 2009 6th Annual IEEE Communications Society Conference on Sensor, Mesh and Ad Hoc Communications and Networks, 2009, pp. 1-9.

[7] Y. Ganjali and A. Keshavarzian, "Load balancing in ad hoc networks: single-path routing vs. multi-path routing," in INFOCOM 2004, vol. 2, 2004, pp. 1120-1125.

[8] Y. Li, M. Pioro, V. Angelakis, D. Yuan, and A. Fragkiadakis, "Mathematical modelling of metric-driven routing and resource allocation in wireless mesh networks," in 16th International Telecommunications Network Strategy and Planning Symposium (Networks), 2014, pp. 1-6.

[9] K. Jain, J. Padhye, V. N. Padmanabhan, and L. Qiu, "Impact of interference on multi-hop wireless network performance," Wireless networks, vol. 11, no. 4, pp. 471-487, 2005.

[10] B. Mumey, J. Tang, and T. Hahn, "Joint stream control and scheduling in multihop wireless networks with MIMO links," in IEEE International Conference on Communications, 2008, pp. 2921-2925.

[11] Y.-H. Lin, T. Javidi, R. L. Cruz, and L. B. Milstein, "Distributed link scheduling, power control and routing for multi-hop wireless MIMO networks," in 2006 Fortieth Asilomar Conference on Signals, Systems and Computers, 2006, pp. 122-126.

[12] S.-J. Kim, X. Wang, and M. Madihian, "Cross-layer design of wireless multihop backhaul networks with multiantenna beamforming," IEEE Transactions on Mobile Computing, vol. 6, no. 11, pp. 1259-1269, 2007.

[13] M. Cao, X. Wang, S.-J. Kim, and M. Madihian, "Multi-hop wireless backhaul networks: A cross-layer design paradigm," IEEE Journal on Selected Areas in Communications, vol. 25, no. 4, pp. 738-748, 2007.

[14] M. Alicherry, R. Bhatia, and L. E. Li, "Joint channel assignment and routing for throughput optimization in multiradio wireless mesh networks," IEEE Journal on Selected Areas in Communications, vol. 24, no. 11, pp. 1960-1971, Nov 2006.

[15] W. Wang, X. Liu, and D. Krishnaswamy, "Robust routing and scheduling in wireless mesh networks under dynamic traffic conditions," IEEE Transactions on Mobile Computing, vol. 8, no. 12, pp. 1705-1717, 2009.

[16] R. A. Iltis, S.-J. Kim, and D. A. Hoang, "Noncooperative iterative mmse beamforming algorithms for ad hoc networks," IEEE Transactions on Communications, vol. 54, no. 4, pp. 748-759, 2006.

[17] M. Johansson and L. Xiao, "Cross-layer optimization of wireless networks using nonlinear column generation," IEEE Transactions on Wireless Communications, vol. 5, no. 2, pp. 435-445, 2006.

[18] S. Boyd and A. Mutapcic, "Subgradient methods," notes for EE364, Standford University, Winter. 2006-07. 